\title{
Changes in Corneal Topography and Clinical Refraction Following Horizontal Rectus Muscle Surgery
}

\author{
Marie Jeazelle H. Redondo, ${ }^{1}$ Alvina Pauline D. Santiago ${ }^{1,2}$ and Ivo John S. Dualan ${ }^{1,3}$ \\ ${ }^{1}$ Department of Ophthalmology and Visual Sciences, College of Medicine and Philippine General Hospital, University of the Philippines Manila \\ ${ }^{2}$ Philippine Eye Research Institute \\ ${ }^{3}$ Eye and Vision Institute, The Medical City
}

\begin{abstract}
Objectives. Refractive changes have been studied after muscle surgery in literature but most results are inconsistent. It has been postulated that changes in corneal tension after muscle surgery may cause a change in corneal curvature resulting in the change in refraction postoperatively. This study investigated changes in corneal topography and clinical refraction after horizontal rectus muscle surgery.

Methods. Twenty-one eyes of 13 patients underwent horizontal rectus muscle surgery via limbal approach. Manifest refraction, cycloplegic refraction, and corneal topography were measured preoperatively, and postoperatively at day 1 and weeks 1, 2, 4 and 8 . The proportion of subjects with at least $0.5 \mathrm{D}$ change from preoperative measurements and the proportion of subjects that needed new prescription postoperative were also computed. Analysis of the results were done using the Friedman test to identify significant differences among measurements at different time periods with post-hoc analysis utilized to identify specific time periods with significant changes from preoperative measurements.

Results. Mean corneal keratometry, horizontal, vertical, and oblique astigmatism, obtained topographically showed no significant difference from preoperative measurements. The statistically significant difference in corneal astigmatism in the recession group at day 1 , week 4 and week 8 postoperatively was not confirmed when converted to power vectors in both vertical/horizontal (J0) and oblique (J45) astigmatism. Clinical refraction showed a transient myopic shift in spherical equivalent, statistically significant only on postoperative day 1 in the recession group. There was no statistically significant difference in clinical astigmatism. There was $\geq 0.5 \mathrm{D}$ change in spherical equivalent in $60 \%$ in both study groups by the end of follow-up. The shift in J0 was more than $10 \%$ in the recession group. More than fifty percent $(52.4 \%)$ needed new prescription for glasses.
\end{abstract}

Conclusion. No statistically significant change in corneal topography and clinical refraction following horizontal rectus muscle surgery were found. Patients should still be refracted at least 2 weeks postoperatively to check if there is a need for change in prescription glasses to improve alignment and/or improve vision.

Key Words: horizontal rectus muscle surgery, rectus recession, rectus resection, changes in refraction, changes in astigmatism, strabismus surgery

$1^{\text {st }}$ place in the Basic Paper Category, 32 $32^{\text {nd }}$ PAO-Novartis Residents' Research Paper Contest, December 4, 2018, SMX Convention Center, Pasay City, Metro Manila.

Presented at the World Society of Pediatric Ophthalmology and Strabismus World Wide Connect, September 26, 2020, virtual meeting.

Corresponding author: Marie Jeazelle H. Redondo, MD

Department of Ophthalmology and Visual Sciences

Philippine General Hospital

University of the Philippines Manila

$5^{\text {th }}$ floor, Chairman's Office,

Sentro Oftalmologico Jose Rizal Building,

Philippine General Hospital,

Taft Avenue, Ermita, Manila 1000, Philippines

Email:mhredondo@up.edu.ph

\section{INTRODUCTION}

Strabismus often begins in childhood and may persist through adulthood, resulting in amblyopia and impaired binocular function unless successfully treated. Strabismus surgery remains the definitive treatment for many cases especially for patients with large deviations. Among the complications of strabismus surgery, changes in refraction postoperatively are rarely given importance. There are conflicting results regarding corneal topography and refraction post-muscle surgery. Some studies showed a transient change in refraction, while others reported a significant change in refraction that became stable postoperatively. ${ }^{1-6}$ Hong and Kang reported a myopic shift and an increase in 
with-the-rule astigmatism, while Preslan et al. reported no significant difference pre- and postoperatively. ${ }^{4,6}$ The exact mechanism for the change in refraction is still unknown but some studies suggest that the change in corneal power and curvature after muscle surgery plays a major role. ${ }^{7,8}$

This study aimed to determine if there was a significant change in both manifest and cycloplegic refraction as well as corneal topography after horizontal rectus muscle surgery. Specifically, the change in mean keratometry reading $(\mathrm{Km})$, topographic and refractive astigmatism, and spherical equivalent after horizontal rectus muscle surgery were determined in order to recommend the optimal time to refract postoperatively.

\section{MATERIALS AND METHODS}

The study utilized a prospective observational cohort study design. Ethics review board approval was obtained from the University of the Philippines Manila Research Ethics Board (UPMREB).

Cooperative patients seven years old or older who were scheduled to undergo horizontal rectus muscle surgery were recruited from the outpatient Pediatric Ophthalmology Clinic of the Department of Ophthalmology and Visual Sciences of the Philippine General Hospital. Horizontal rectus muscle procedures were in the form of recession, resection, or recession and resection (R\&R) and posterior fixation suture placement (fadenoperation). Patients who have irregular cornea (e.g., corneal scar, keratoconus), pterygium and other diseases affecting the cornea, Graves disease, orbital myositis, Duane syndrome, Moebius syndrome, retinal detachment and other diseases directly affecting the extraocular muscle/s (e.g., ocular myositis, ocular myasthenia) were excluded from this study. Other exclusion criteria include previous strabismus surgery, other types of muscle surgery with manipulation of other muscles other than the horizontal rectus muscle/s, and patients unable to follow instructions for completion of corneal topography examination.

Twenty-three eyes of 13 patients were enrolled in this study, and stratified into groups depending on the type of strabismus surgery: recession (11), recession and resection (10), resection (1), and fadenoperation (1). Measurements of best-corrected visual acuity, ocular alignment, corneal topography using the Oculus Keratograph, manifest refraction and cycloplegic refraction using handheld retinoscopy were taken 1-2 weeks preoperatively and postoperatively at 1 day, 1 week, 2 weeks, 4 weeks and 8 weeks by a single investigator. Cycloplegic refraction was performed by instilling a combination of tropicamide $0.5 \%$ and phenylephrine $0.5 \%$ eye drops (Sanmyd-P), 1 drop every 3 to 5 minutes on both eyes for 3 doses. Retinoscopy was performed 30 minutes after the initial drop. Only cycloplegic refraction was presented in the results because not all patients had manifest refraction.

All surgeries were done at the Department of Ophthalmology and Visual Sciences Philippine General
Hospital. The surgeries followed standard procedures described by Parks for recession and resection done singly or together (Appendices 1 and 2). ${ }^{9,10}$ The amount of muscle recessed or resected were based on Parks' recommended surgical numbers based on preoperative angle of deviation. All surgeries involved dissection of the conjunctiva via limbal approach with relaxing cuts at oblique quadrant/s. ${ }^{11}$ Placement of a posterior fixation suture (fadenoperation) may be included with or without recession. ${ }^{12}$

\section{Corneal topography}

Mean change in corneal power $(\mathrm{Km})$ and astigmatism from the corneal topography results were analyzed by computing for the absolute value of the difference between the pre- and post-operative measurements. Topographic astigmatism results were analysed using cylinder power alone, as well as recomputed using the power vector analysis.

\section{Power vector analysis for astigmatism}

In the analysis of astigmatic change, the power of the cylinder or astigmatism was converted to the power vector notation to account for the directional effect of the astigmatism while simultaneously allowing precise statistical analysis of the astigmatic axis. ${ }^{4,5}$

Astigmatism was converted to power vector notation using Miller's formula:

$$
\begin{gathered}
J 0=-\frac{(C+0.000001)}{2} \times \cos \left(\frac{A \times 2 \times P I}{180}\right) \\
J 45=-\frac{(C+0.000001)}{2} \times \sin \left(\frac{A \times 2 \times P I}{180}\right)
\end{gathered}
$$

where $C$ is the cylinder in minus cylinder format, $\mathrm{A}$ is the cylindrical axis. ${ }^{13} \mathrm{~J} 0$ represents the vertical astigmatism at the $90^{\circ}$ and $180^{\circ}$ meridians, where positive values of $\mathrm{J} 0$ indicate with-the-rule astigmatism and negative values indicate against-the-rule astigmatism. On the other hand, J45 represents oblique astigmatism.

Clinical refraction results were shown as spherical equivalents and using the power vector analysis for astigmatism as described above. The change in refraction was computed using the absolute value of the difference between pre- and postoperative manifest and cycloplegic refraction.

\section{Spherical equivalent}

The pre- and postoperative spherical equivalent were computed using the traditional formula:

$$
\text { Spherical equivalent }=S+\frac{1}{2} C
$$

As well as Miller's formula:

$$
\text { Spherical equivalent }=+\left(S+\frac{C+0.00001}{2}\right)
$$

where $\mathrm{S}$ is the sphere (diopter) and $\mathrm{C}$ is the cylinder in minus format. 
Proportion of patients with at least $0.5 \mathrm{D}$ change from preoperative measurements

The proportion of subjects with $\geq 0.5 \mathrm{D}$ change was measured by counting the total number of eyes that had at least $0.5 \mathrm{D}$ difference from the preoperative measurements in $\mathrm{Km}$, corneal topographic astigmatism, clinical astigmatism and spherical equivalent. A change of at least $0.5 \mathrm{D}$ was considered clinically significant as this may translate to a change in visual acuity and/or alignment postoperatively., 2,5

\section{Need for change in prescription}

The proportion of subjects that needed a change in prescription glasses postoperatively was calculated by getting the total number of eyes that needed change in refraction (at any time during the follow-up period) divided by the total number of eyes enrolled in the study. Reason for the change in prescription may be for improvement of visual acuity or improvement of the residual deviation.

\section{Statistical analysis}

Baseline data were compared using paired t-test analysis. Categorical variables were compared using the Fisher's exact analysis to determine significant differences among the baseline parameters. Nonparametric method was used for analyzing refraction based on ranking, rather than its actual value. A two-step approach was employed in analyzing the data. Friedman test was used to identify significant differences among measurements at different time periods $(p<0.05)$. When $\mathrm{p}<0.05$, a post hoc analysis was performed to identify the specific time period that was significantly different from preoperative values.

\section{RESULTS}

Twenty-three eyes of 13 patients were enrolled in this study. Of the 23 eyes, only 21 were analyzed-10 underwent monocular recession, 1 monocular recession with fadenoperation, and 10 monocular recession and resection. The 2 subjects who were not included in the analysis were 1 who underwent monocular resection, and 1 who underwent fadenoperation alone. These 2 were excluded because only 1 subject was enrolled per type of strabismus surgery.

The baseline characteristics between the two groups were mostly comparable (Table 1 ). Despite a statistically significant difference in the target deviation between the two groups ( $p=0.0028)$, the amount of recession was nonetheless comparable $(\mathrm{p}=0.0847)$. All other parameters showed comparable characteristics in both groups.

\section{Corneal topography changes (Table 2)}

\section{Change in mean keratometry}

The change in mean keratometry was not statistically significant for both the recession and the R\&R group. It was observed, however, that there was an initial steepening until week 1 , followed by subsequent flattening in the recession

Table 1. Baseline Characteristics of Subjects

\begin{tabular}{lccc}
\multicolumn{1}{c}{$\begin{array}{c}\text { Baseline } \\
\text { characteristics }\end{array}$} & $\begin{array}{c}\text { Recession } \\
\mathbf{N}=11 ; \mathbf{n}(\%)\end{array}$ & $\begin{array}{c}\text { Recession and } \\
\text { Resection } \\
\mathbf{N}=10 ; \mathbf{n}(\%)\end{array}$ & p-value \\
\hline $\begin{array}{l}\text { Age (years) } \\
\text { Sex }\end{array}$ & $23.72 \pm 17.31$ & $20.60 \pm 9.13$ & 0.6162 \\
$\quad$ Female & $9(81.82)$ & $8(80.00)$ & 0.6690 \\
$\quad$ Male & $2(18.18)$ & $2(20.00)$ & \\
Laterality & $5(45.45)$ & $4(40.00)$ & 0.5750 \\
$\quad$ OD & $6(54.55)$ & $6(60.00)$ & \\
$\quad$ OS & $9(42.86)$ & $5(23.81)$ & Not Tested \\
Esotropia & $2(9.52)$ & $5(23.81)$ & \\
Exotropia & $41.82 \pm 12.90$ & $66.11 \pm 18.50$ & $0.0028^{*}$ \\
Target deviation (PD) & & & \\
Amount resected (mm) & Not Applicable & $7.50 \pm 1.17$ & Not Tested \\
Amount recessed (mm) & $7.50 \pm 1.17$ & $6.69 \pm 1.22$ & 0.0847 \\
\hline
\end{tabular}

Note: statistically significant $p$-values $<0.05$ marked with asterisk $(*)$.

Table 2. Mean Corneal Keratometry Reading (Km), Corneal Astigmatism or Cylinder, and Its Vertical (J0) and Oblique (J45) Astigmatic Power Vector Equivalent at Different Follow-up Periods, Mean (SD)

\begin{tabular}{|c|c|c|c|c|c|c|c|}
\hline & Preoperative & Day 1 & Week 1 & Week 2 & Week 4 & Week 8 & p-value \\
\hline \multicolumn{8}{|c|}{ Recession (in diopters, $D$ ) } \\
\hline $\mathrm{Km}$ & $43.13(1.66)$ & $43.20(2.57)$ & $43.33(1.59)$ & $43.21(1.81)$ & $43.09(1.84)$ & $43.25(1.76)$ & 0.952 \\
\hline Cylinder & $-0.95(0.38)$ & $-1.50(0.75)$ & $-1.02(0.41)$ & $-1.05(0.46)$ & $-1.29(0.55)$ & $-1.42(0.51)$ & $<0.001^{*}$ \\
\hline$p$-value & & $0.003^{*}$ & 0.523 & 0.199 & $0.005^{*}$ & $0.008^{*}$ & \\
\hline jo & $0.43(0.17)$ & $0.65(0.39)$ & $0.46(0.19)$ & $0.46(0.23)$ & $0.61(0.28)$ & $0.65(0.24)$ & 0.704 \\
\hline J45 & $0.15(0.14)$ & $0.28(0.24)$ & $0.16(0.16)$ & $0.19(0.17)$ & $0.17(0.12)$ & $0.22(0.20)$ & 0.183 \\
\hline \multicolumn{8}{|c|}{ Recession and Resection (in diopters, D) } \\
\hline $\mathrm{Km}$ & $44.18(1.50)$ & $44.12(1.51)$ & $44.05(1.62)$ & $44.16(1.73)$ & $44.18(1.58)$ & $44.27(1.67)$ & 0.587 \\
\hline Cylinder & - $1.03(0.37)$ & - $1.77(0.86)$ & $-1.53(0.55)$ & - $1.18(0.26)$ & - $1.06(0.45)$ & - $1.32(0.39)$ & 0.050 \\
\hline JO & $0.43(0.19)$ & $0.78(0.45)$ & $0.58(0.39)$ & $0.50(0.18)$ & $0.47(0.22)$ & $0.58(0.22)$ & 0.798 \\
\hline J4 & $0.25(0.13)$ & $0.35(0.21)$ & $0.28(0.17)$ & $0.28(0.13)$ & $0.21(0.14)$ & $0.26(0.13)$ & 0.390 \\
\hline
\end{tabular}

Note: Diopter, D; vertical astigmatism (JO) at $90^{\circ}$ and $180^{\circ}$ calculated using Miller's formula [positive values represent with-the-rule astigmatism and negative values represent against-the-rule astigmatism]; oblique astigmatism (J45) calculated using Miller's formula; standard deviation, SD; statistically significant $p$-values $<0.05$ marked with asterisk (*). 
group. In contrast, an opposite trend was seen in the R\&R group where an initial flattening was documented until week 1 , with note of subsequent steepening from week 2 onwards. In both groups, the trend was for $\mathrm{Km}$ to revert back to preoperative measurements.

\section{Change in astigmatism}

The change in corneal astigmatism (when axis of astigmatism was disregarded) from corneal topography readings revealed a statistically significant difference in the recession group at day 1 , week 4 and week 8 postoperatively. Upon conversion to its power vector equivalent, however, there was no statistically significant change in the vertical (J0) and oblique (J45) astigmatism in the recession group. For the R\&R group, no statistically significant difference in the cylinder, as well power vector equivalents J0 J45 were found.

\section{Clinical refraction (Table 3)}

\section{Spherical equivalent}

There was a transient statistically significant myopic shift at day 1 in the recession group that decreased over time. This trend was also evident in the R\&R group, however, the change was not statistically significant.

\section{Clinical refraction}

Based on clinical refraction, there was an initial increase in with-the-rule astigmatism at day 1 post-op, but this immediately reverted back to preoperative measurements by week 1 and became stable until week 8-this mimicked the trend observed in astigmatism from corneal topography. Using the power vector analysis, the change from preoperative J0 decreased over time. This trend was observed for both recession and R\&R group but was not statistically significant. No oblique astigmatism was seen in the recession group. Some oblique astigmatism was observed in the R\&R group but this was not statistically significant.

\section{Proportion of patients with at least $0.5 \mathrm{D}$ change from preoperative measurements}

\section{Corneal topography change in $\mathrm{Km}$}

The proportion of eyes with $\geq 0.5 \mathrm{D}$ change in mean keratometry reading $(\mathrm{Km})$ by the end of follow-up was $30 \%$ in the recession group and $10 \%$ in the $\mathrm{R} \& \mathrm{R}$ group (Figures $1 \mathrm{~A}$ and $1 \mathrm{~B}$ ).

\section{Corneal topography change in astigmatism}

Ignoring the cylinder axis, the recession group had $40 \%$ showing $\geq 0.5 \mathrm{D}$ change in corneal astigmatism whereas $30 \%$ were observed in the R\&R group by the end of follow-up (Figures $1 \mathrm{C}$ and $1 \mathrm{D}$ ). In contrast, conversion to power vector equivalent decreased the proportion of $\geq 0.5 \mathrm{D}$ change in vertical astigmatism to $10 \%$ in the recession group, and nil in the R\&R by the end of 2 months(Figures $1 \mathrm{E}$ and $1 \mathrm{~F}$ ). There were no topographically significant changes in oblique astigmatism in both groups (Figures $1 \mathrm{G}$ and $1 \mathrm{H}$ ).

\section{Changes in clinical refraction}

Sixty percent $(60 \%)$ had $\geq 0.5 \mathrm{D}$ change in spherical equivalents in both the recession and R\&R group by the end of follow-up (Figures 2A and 2B).

Figure $2 \mathrm{C}$ shows that there was only $9 \% \geq 0.5 \mathrm{D}$ change in $\mathrm{J} 0$ at day 1 , immediately disappearing by week 1 onwards. In the R\&R group, $10 \%$ had $\geq 0.5 \mathrm{D}$ change in J0 by week 8 (Figure 2D). Similar to topographic oblique astigmatism, there was absence of $\geq 0.5 \mathrm{D}$ change in J45 or oblique astigmatism in both groups (Figures $2 \mathrm{E}$ and $2 \mathrm{~F}$ ).

\section{Need for prescription glasses}

Eleven of 21 subjects (52.4\%) required new prescription glasses, given starting from 2 weeks to 2 months postoperatively. The reason for the new prescription was presence of residual deviation that could be helped by wearing of a new prescription and was not due to any reduction in postoperative visual acuity.

Table 3. Mean Change in Spherical Equivalent (SE) and Astigmatism (JO and J45) Following Clinical Refraction at Different Follow-up Periods, Mean (SD)

\begin{tabular}{|c|c|c|c|c|c|c|c|}
\hline & Preoperative & Day 1 & Week 1 & Week 2 & Week 4 & Week 8 & p-value \\
\hline \multicolumn{8}{|c|}{ Recession (in diopters, $D$ ) } \\
\hline SE & $1.44(0.43)$ & $1.03(0.42)$ & $1.14(0.38)$ & $1.23(0.26)$ & $1.33(0.46)$ & $1.38(0.27)$ & $0.010^{*}$ \\
\hline$p$-value & & $0.049^{*}$ & 0.056 & 0.131 & 0.705 & 0.916 & \\
\hline JO & $0.06(0.12)$ & $0.15(0.28)$ & $0.05(0.10)$ & $0.05(0.15)$ & $0.05(0.12)$ & $0.02(0.08)$ & 0.460 \\
\hline J45 & $0.00(0.00)$ & $0.00(0.00)$ & $0.00(0.00)$ & $0.00(0.00)$ & $0.00(0.00)$ & $0.00(0.00)$ & 0.080 \\
\hline \multicolumn{8}{|c|}{ Recession and Resection (in diopters, D) } \\
\hline SE & $0.91(0.77)$ & $0.85(0.56)$ & $0.79(0.84)$ & $0.74(0.72)$ & $0.70(0.72)$ & $0.88(0.70)$ & 0.490 \\
\hline JO & $0.21(0.48)$ & $0.49(0.23)$ & $0.31(0.29)$ & $0.21(0.29)$ & $0.22(0.25)$ & $0.17(0.23)$ & 0.120 \\
\hline $\mathrm{J} 4$ & $0.00(0.00)$ & $0.04(0.08)$ & $0.01(0.04)$ & $0.01(0.04)$ & $0.02(0.05)$ & $0.02(0.05)$ & 0.510 \\
\hline
\end{tabular}

Note: Diopter, D; vertical astigmatism (JO) at 900 and $180 \mathrm{o}$ calculated using Miller's formula [positive values represent with-the-rule astigmatism and negative values represent against-the-rule astigmatism]; oblique astigmatism (J45) calculated using Miller's formula; standard deviation, SD; statistically significant $p$-values $<0.05$ marked with asterisk (*). 


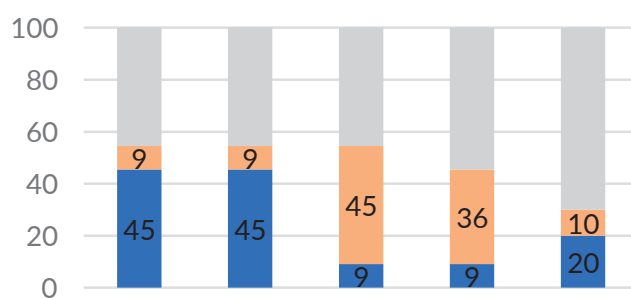

A day 1 week 1 week 2 week 4 week 8
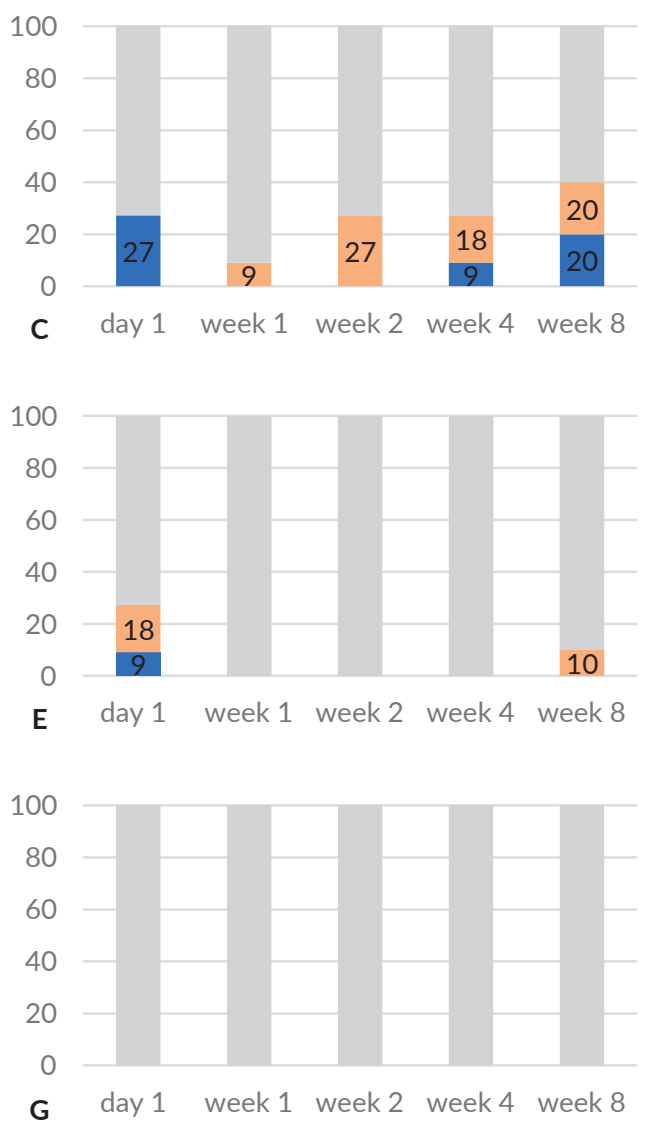

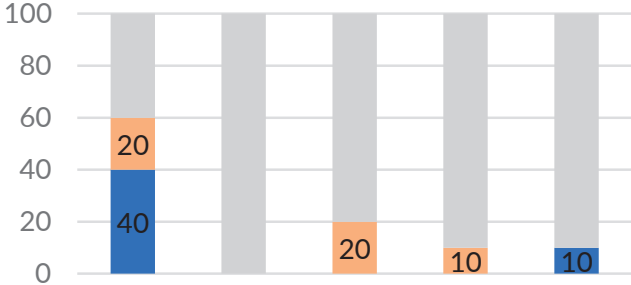

B day 1 week 1 week 2 week 4 week 8
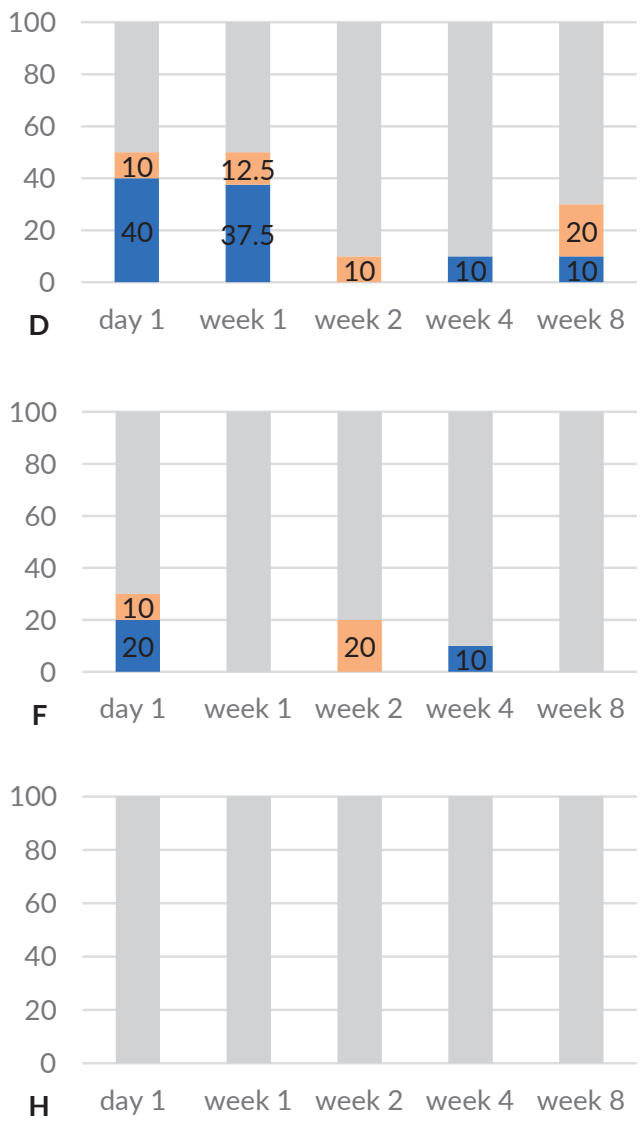

H day 1 week 1 week 2 week 4 week 8

Figure Legends: $\quad \square>0.75$

$0.5-0.75$

$<0.5$

Figure 1. Proportion of eyes with $\geq 0.5 \mathrm{D}$ change in mean keratometry $(\mathrm{Km})$, astigmatism, and power vector equivalent in the vertical (JO) and oblique (J45) meridian based on corneal topography. (A) values for Km for the recession group; (B) values for Km for the R\&R group; (C) values for corneal astigmatism disregarding axis of the cylinder for the recession group; (D) values for the R\&R group; (E) proportion with $\geq 0.5 \mathrm{D}$ change in J0 for the recession group; (F) values for the R\&R group; (G) proportion with $\geq 0.5 \mathrm{D}$ change in $\mathrm{J45}$ for the recession group; $(\mathrm{H})$ values for the R\&R group.

\section{DISCUSSION}

Corneal topography results showed no significant change in the mean keratometry $(\mathrm{Km})$ readings from preoperative values for all horizontal rectus muscle surgeries, namely recession with or without fadenoperation, and recession and resection (R\&R). This was consistent with the study by Preslan et al. ( $\mathrm{n}=107$ eyes), wherein they found no significant difference in the corneal topography obtained 4 months post-operatively. ${ }^{6}$
Recession showed an initial corneal steepening up to 1 month postoperatively followed by subsequent corneal flattening in the subsequent follow-up. This was consistent with Cakmak et al. who documented an initial steepening up to 1 month postoperatively, then decreased until the $3^{\text {rd }}$ month postoperatively. ${ }^{14}$ However, in their study, they found a statistically significant surgically induced astigmatism in recession, which was higher in the group operated on by hang-back technique compared to the conventional recession technique. This steepening in recession was also consistent with the inferior steepening found in inferior 

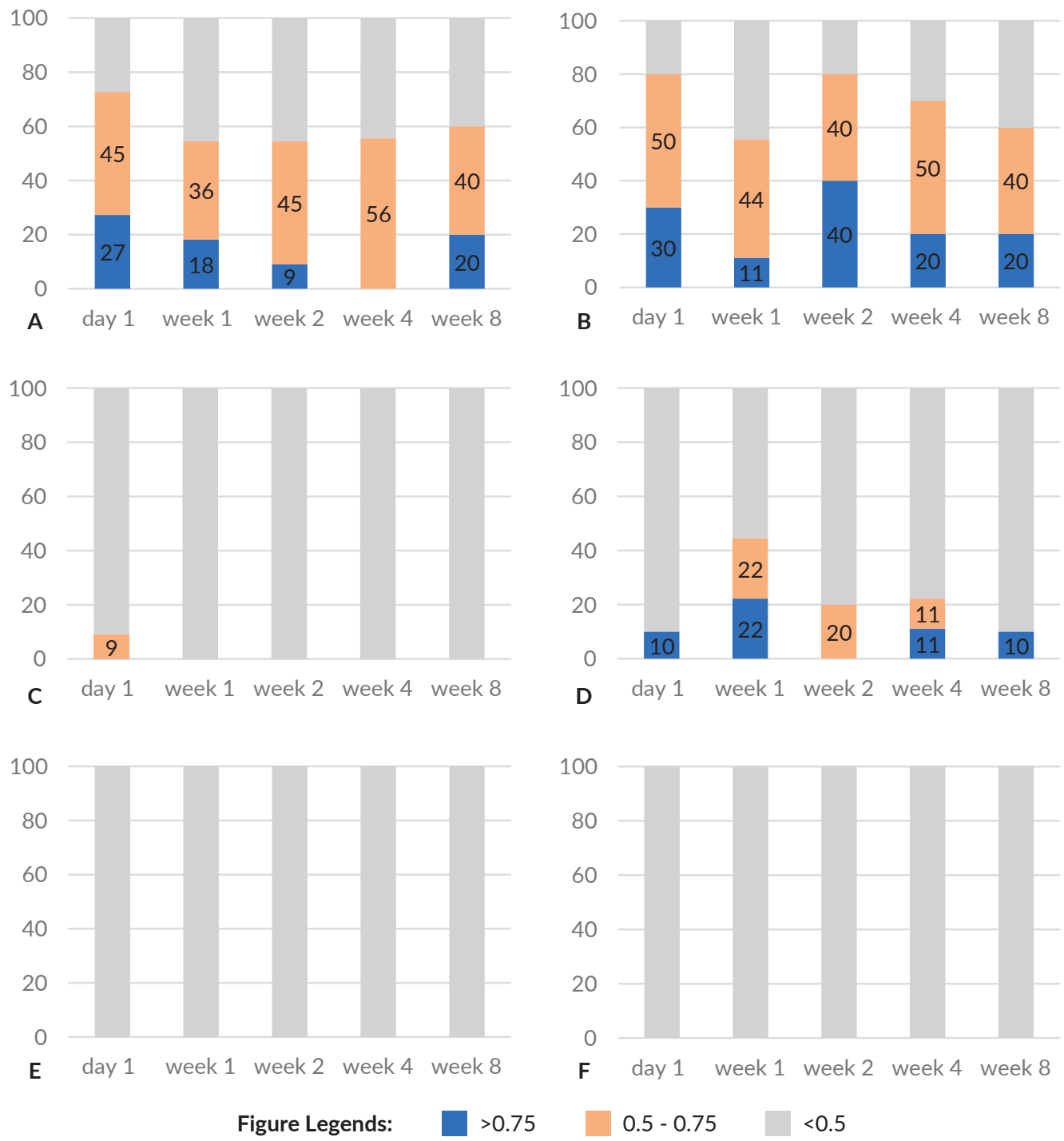

Figure 2. Proportion of eyes with $\geq 0.5 \mathrm{D}$ change in spherical equivalent and astigmatism based on clinical refraction. (A) proportion of eyes that showed $\geq 0.5 \mathrm{D}$ change in spherical equivalent for the recession group; (B) values for the R\&R group; (C) proportion with changes in J0 in the recession group; (D) values for the R\&R group; (E) proportion that showed $\geq 0.5 \mathrm{D}$ change in $\mathrm{J45}$ for the recession group; (F) values for the R\&R group. J0, vertical astigmatism; J45, oblique astigmatism, calculated using Miller's formula.

rectus recession of Graves' disease patients. ${ }^{8}$ This was also seen by Bae and Choi during the $1^{\text {st }}$ week postoperative follow-up, but measurements reverted back to original after 3 months. ${ }^{15}$

Recession and resection showed an initial corneal flattening until 1 month postoperatively, followed by subsequent corneal steepening. This flattening could have been induced by the resection of the muscle. This was consistent with the study by Hegazy et al., wherein they found an initial corneal flattening at 3 months postoperatively, but was statistically insignificant. ${ }^{16}$ Bae and Choi also found no statistically significant difference in mean corneal keratometry values in their R\&R group. ${ }^{15}$

In the analysis of the cylinder alone, without consideration of its axis, there was significant difference at day
1 , week 4 and week 8 in the recession group. However, upon conversion to the power vector equivalent, there were no significant differences in all postoperative follow-up period. This result suggests that conversion to power vector provides a more accurate measure of astigmatism which can be compared and subjected to statistical analysis, whereas disregarding the axis can lead to overestimation of the cylindrical value.

Similar to other studies, there was no significant difference in vertical and oblique astigmatism in our study. ${ }^{3,6}$

We found a significant transient myopic shift in the recession group, and a tendency towards with-the-rule astigmatism, similar to studies by Hong and Kang, and Hegazy et al.,16 However, Hong and Kang reported a stable decrease in spherical equivalent up to six months postoperatively and stable change in astigmatism up to 3 
months postoperatively. ${ }^{4}$ The dissimilarities in the results could have been brought about by the larger sample size and difference in method of obtaining refraction in Hong and Kang study that measured noncycloplegic refraction using an autorefractometer in patients who underwent limbal incision horizontal rectus muscle surgery. ${ }^{4}$ Our study used cycloplegic refraction as the basis of statistical analysis.

We also looked at the proportion of eyes with $\geq 0.5 \mathrm{D}$ change in refraction postoperatively. Only $10 \%$ of the R\&R group ( $n=10)$ had $\geq 0.5 \mathrm{D}$ change in cylinder by the end of the 2-month follow-up period. Comparing the results of this study with that by Mezad-Koursh et al., the latter found that $45.2 \%$ eyes ( $n=31$ eyes) had $\geq 0.5 \mathrm{D}$ change in cylinder at 1 month postoperatively. ${ }^{5}$ In contrast, Nardi et al. found only transient and insignificant change in astigmatism, and that the residual astigmatism of $>0.5 \mathrm{D}$ at 30 days postoperatively was found in only $12 \%$ ( $n=52$ eyes) of their patients. ${ }^{2}$

In both recession and R\&R groups, $60 \%$ had $\geq 0.5 \mathrm{D}$ change in spherical equivalent at 2 months postoperatively. In Mezad-Koursh et al. study, only $32.3 \%$ had $\geq 0.5 \mathrm{D}$ change in spherical equivalent. ${ }^{5}$

In our study, 11 of the 21 eyes (52.4\%) needed new prescription for glasses postoperatively, prescribed at least 2 weeks to 2 months postoperatively. In contrast, only $35.5 \%$ ( $\mathrm{n}=31$ eyes) needed new prescription at 1 month postoperatively in the study by Mezad-Koursh et al. ${ }^{5}$ The main indication for the change in prescription glasses in our study was to correct less than optimal residual deviation (more than $8 \mathrm{PD}$ ).

The etiology of the change in refraction after horizontal rectus muscle surgery has not been fully understood. Theories implicate changes in muscle tension postoperatively as the basis or major contributor to changes observed in corneal topography. $1,7,8$ The absence of a significant difference in refractive changes could also be explained by "coupling" of the corneal quadrants wherein corneal curvature changes in one quadrant are offset by reciprocal changes in other quadrants, resulting to maintenance of a similar astigmatism. ${ }^{8,17}$ Other studies postulated a role contributed by a change in lenticular astigmatism as being responsible for causing a shift in refraction but this was not included in the scope of this study. ${ }^{6}$ Relative anterior segment ischemia following disruption of ciliary circulation after muscle surgery has been postulated as the cause but studies to prove this theory remain wanting. ${ }^{6}$

As recommendations, to reduce the confounding factor contributed by different surgeons with different techniques, it would have been ideal that all horizontal rectus surgeries be performed by a single surgeon. Unfortunately, this could not be adhered to in our setting as the study was carried out in a training institution with different operating surgeons in training. Using a set of retinoscopy rack, with results confirmed with trial lenses on a trial frame are likewise recommended. This avoids instrument convergence that could be contributed by phoropter use. Corneal topography should be obtained prior to instillation of any eye drops, since this may be a confounding factor in obtaining keratometric measurements. Evidence is also always stronger with larger sample size, so increasing recruitment of more patients for a similar study is suggested.

\section{CONCLUSION}

Overall, change in refraction is not clinically significant whether topographically or based on manifest and cycloplegic refraction. Transient change observed in the early postoperative period reverts back close to pre-operative levels in most cases. Our clinical recommendation is still to do refraction at least 2 weeks postoperatively, then repeat the refraction at 2 months postoperatively, especially for cases with either residual deviation or reduction in visual acuity that may contribute to successful alignment or development of amblyopia.

\section{Acknowledgments}

I would like to thank my co-investigators, Dr. Pauline Santiago and Dr. Ivo Dualan for their comments, support and advise throughout the study. Our statistician, Ms. Caren Joy Bacsid, thank you. We would like to thank the Department, our Chair Dr. Marissa Valbuena and our Vice Chair for Research Dr. Teresita Castillo for believing in our study. Thank you to the love of my life, Dr. Roel Villanueva. And for all those who have in some way contributed to the conception, conduction and analysis of the study, our deepest gratitude to all of you.

\section{Statement of Authorship}

All authors participated in data collection and analysis, and approved the final version submitted.

\section{Author Disclosure}

The authors declared no conflict of interest relevant to the conduct of the study.

\section{Funding Source}

The Department of Ophthalmology and Visual Sciences waived fees for Oculus Keratograph.

\section{REFERENCES}

1. Marshall D. Changes in refraction following operation for strabismus. Arch Ophthalmol. 1936;15(6):1020-31. doi:10.1001/ archopht.1936.00840180064006.

2. Nardi M, Rizzo S, Pellegrini G, Lepri A. Effects of strabismus surgery on corneal topography. J Pediatr Ophthalmol Strabismus. 1997 JulAug; 34(4):244-6.

3. Kitthaweesin K, Singhakul S. Effect of horizontal strabismus surgery on the astigmatism. J Med Assoc Thai. 2007 Apr; 90(4):744-7.

4. Hong SW, Kang NY. Astigmatic changes after horizontal rectus muscle surgery in intermittent exotropia. Korean J Ophthalmol. 2012 Dec; 26(6):438-45. doi:10.3341/kjo.2012.26.6.438.

5. Mezad-Koursh DM, Leshno A, Ziv-Baran T, Stolovitch C. Refractive changes induced by strabismus corrective surgery in adults. J Ophthalmol. 2017; 2017:2680204. doi: 10.1155/2017/2680204. 
6. Preslan MW, Cioffi G, YI Min. Refractive error changes following strabismus surgery. J Pediatr Ophthalmol Strabismus. 1992 SepOct; 29(5):300-4. doi: 10.3928/0191-3913-19920901-09.

7. Hainsworth DP, Bierly JR, Schmeisser ET, Baker RS. Corneal topographic changes after extraocular muscle surgery. J AAPOS. 1999 Apr; 3(2):80-6.

8. Kwitko S, Feldon S, McDonnell PJ. Corneal Topographic Changes Following Strabismus Surgery in Graves' Disease. Cornea. 1992 Jan; 11(1):36-40.

9. Parks MM. Atlas of strabismus surgery. New York: Harper \& Row; 1983.

10. Helveston E. Surgical Management of Strabismus, 5th ed. Oostende: Wayenborgh; 2005. Available from: https://www. aao.org/Assets/86858597-4bc8-4e9b-a94f-8960daf40cb0/ $636343503861030000 /$ strabismus-surgical-management-ofstrabismus-ehelveston-pdf?inline $=1$.

11. Sami DA. Conjunctival incisions for strabismus surgery: a comparison of techniques. Techniques in Ophthalmology. 2007; 5(3):125-9.

12. Kaufmann H. Results of Cüppers Fadenoperation (Posterior Fixation Suture). In: van Balen ATM, Houtman WA, eds. Strabismus Symposium Amsterdam, September 3-4, 1981. Documenta Ophthalmologica Proceedings Series, vol 32. Springer, Dordrecht. 1982. https://doi.org/10.1007/978-94-009-7997-0_23

\section{APPENDICES}

\section{Appendix 1. Standard Procedure for Recession 9,10}

The rectus muscle is identified and dissected from the surrounding intermuscular septum with sharp dissection. Pre-placement of a double-armed 6-0 synthetic, absorbable suture (Vicryl 5-0 or 6-0) with spatulated needle (S-24 or $\mathrm{S}-19)$ is done $1 \mathrm{~mm}$ from the muscle's insertion to sclera. The suture is secured with a square knot in the middle of the muscle about $1 \mathrm{~mm}$ from the sclera. The needle is then passed in the substance of the muscle going towards the lateral end of the muscle. The needle is passed perpendicularly through the tendon to include only $1 \mathrm{~mm}$ to $1 / 3$ of the width of the muscle in the locking bite. Pre-placement of suture is completed by placing the inferior half of the suture from the point at which the superior half has left off. Similarly, the suture is placed $1 \mathrm{~mm}$ from the insertion of the tendon into the sclera. The needle is likewise passed perpendicularly through the tendon to include only $1 \mathrm{~mm}$ to $1 / 3$ of the width of the muscle in the locking bite. Hemostasis prior to disinsertion is performed. While applying an upward tension on the preplaced suture and holding the muscle taut with a muscle hook, disinsertion proceeds by cutting the tendon flush to the sclera. The sclera is then grasped at the area of the residual muscle stump to expose the site of scleral entry. The desired recession is measured from the area where the muscle used to be inserted using a caliper. The needles are then be inserted through the sclera entering the tissue at the marked area. Finally, the sutures are tied and cut. The new insertion should be inspected before conjunctiva is closed. The conjunctiva may be closed using the same vicryl sutures or with smaller caliber such as 7-0 or 8-0 absorbable sutures. Should chromic (or catgut) sutures be available, this absorbs
13. Miller JM. Clinical applications of power vectors. Optom Vis Sci. 2009 Jun; 86(6):599-602.

14. Cakmak H, Kocaturk T, Dundar SO. Comparison of surgically induced astigmatism in patients with horizontal rectus muscle recession. Int J Ophthalmol. 2014 Aug; 7(4):709-13. doi:10.3980/j.ssn.2223959.2014.04.23.

15. Bae SH, Choi DG. Changes of corneal topographic measurements and higher-order aberrations after surgery for exotropia. PLoS One. 2018 Aug; 13(8):e0202864. doi: 10.1371/journal.pone.0202864.

16. Hegazy HS, Lamie NM, Abd El Salam RH, Effect of strabismus surgery on refractive power of the eye. Al-Azhar Assiut Medical Journal. 2018; 16(2):141-7. doi: 10.4103/AZMJ.AZMJ_40_18.

17. Kwito S, Sawusch MR, McDonnell PJ, Gritz DC, Moreira H, Evensen D. Effect of extraocular muscle surgery on corneal topography. Arch Ophthalmol. 1991 Jun; 109(6):873-8.

faster and will preclude prolonged conjunctival inflammation or redness. If the conjunctiva is tight, conjunctiva may be anchored at a slightly recessed site.

\section{Appendix 2. Standard Procedure for Resection 9,10}

Resection is started by identifying, dissecting and exposing the rectus muscle. The amount of resection is measured from the muscle insertion. A single assistant exposes the rectus muscle using 2 muscle hooks held just taut. Pre-placement of the suture in the rectus muscle is done using one double-armed synthetic absorbable suture (Vicryl 5-0 or 6-0) with spatulated needle (S24 or S19). The suture is anchored in the middle of the muscle belly where the caliper marks the amount of resection. The superior half of the suture will enter the muscle belly and exit the lateral end of the muscle. The needle will then be passed perpendicularly through the tendon to include only $1 \mathrm{~mm}$ to $1 / 3$ of the width of the muscle in the locking bite. Pre-placement of the suture is completed by placing the inferior half of the suture from the point at which the superior half has left off proceeding in the same manner as the superior half. Hartman forceps or hemostat is placed $1 \mathrm{~mm}$ distal to the sutures for hemostasis. The muscle is cut where the forceps used to clamp the muscle. The resected muscle is inspected making sure the sutures are secure. The muscle stump anterior to the area is resected and removed from its stump. If residual muscle at the stump is desired, Westcott scissors are held at a slight angle. If the desire is to cut flushed to the insertion, the blades of the scissors are held parallel to the scleral plane. The needle is then passed through the muscle stump at the insertion. Conjunctiva is closed primarily using absorbable sutures as described in recession. 
Appendix 3. Drug information ${ }^{16}$

Drug Name: Sanmyd-P

Manufacturer: Santen

Distributor: Croma Medic Inc

Contents: Tropicamide $0.5 \%$, Phenylephrine Hydrochloride $0.5 \%(\mathrm{w} / \mathrm{v})$

Indications: Mydriasis \& cycloplegia for refraction

Dosage: For cycloplegia, instill 1 drop 2-3 times every 3-5 minutes allowing examination 30 minutes after

Contraindications:

This product is contraindicated in the following patients:

1. Patients with glaucoma or those predisposed to ocular hypertension as evidenced by a narrow angle or shallow anterior chamber (Acute angle closure glaucoma may occur)

2. Patients with a history of hypersensitivity to any ingredients of this product.

Special precautions:

Careful administration in the following patients:

1. Infants: Administer cautiously when applied to premature infants under close observation of patient's condition since it has been shown to cause bradycardia or apnea.
2. Hypertensive patients

3. Patients with arteriosclerosis

4. Patients with heart disease, including coronary artery disease or heart failure

5. Patients with diabetes

6. Patients with hyperthyroidism

Adverse Drug Reactions:

1. Clinically significant adverse reactions (rarely $<0.1 \%$, occasionally between 0.1 and $5 \%$ )

2. Shock, anaphylactoid reaction

3. Hypersensitivity: (blepharitis, eyelid dermatitis, itching, rash, urticaria)

4. Ocular: conjunctivitis, corneal epithelium disorder, rise of intraocular pressure

5. Gastrointestinal: thirst, nausea/vomiting

6. Others: facial flushing, tachycardia, hypertension, headache

MIMS Class: Mydriatic Drugs

ATC Classification: S01FA06 - tropicamide ; Belongs to the class of anticholinergics used as mydriatics and cycloplegics. Regulatory Classification: $\mathrm{Rx}$

Packaging/Price: 15 mL x 1's (Php 684.00/ container) 
Appendix 4. Raw data and statistical analysis

Table 4. Median values of preoperative and postoperative manifest refraction

\begin{tabular}{|c|c|c|c|c|c|c|c|}
\hline & Preoperative & Day 1 & Week 1 & Week 2 & Week 4 & Week 8 & p-value \\
\hline \multicolumn{8}{|c|}{ Recession (in diopters, D) } \\
\hline SE & 1.000 & 0 & 1.000 & 0.750 & 0.630 & 1.315 & 0.049 \\
\hline J0 & 0 & 0 & 0 & 0 & 0 & 0 & 0.075 \\
\hline J45 & 0 & 0 & 0 & 0 & 0 & 0 & 0.075 \\
\hline \multicolumn{8}{|c|}{ Recession and Resection (in diopters, D) } \\
\hline SE & -0.250 & -0.750 & -0.880 & -1.250 & -0.750 & -0.750 & 0.216 \\
\hline JO & 0 & 0 & 0 & 0 & 0 & 0 & 0.774 \\
\hline $\mathrm{J} 45$ & 0 & 0 & 0 & 0 & 0 & 0 & 0.512 \\
\hline
\end{tabular}

Table 5. Median values of preoperative and postoperative cycloplegic refraction

\begin{tabular}{lrrrrrr}
\multicolumn{2}{c}{ Preoperative } & Day 1 & Week 1 & Week 2 & Week 4 & Week 8 \\
Recession (in diopters, D) & & & & & \\
SE & 1.500 & 1.000 & 1.250 & 1.000 & 1.500 & 1.500 \\
J0 & 0 & 0 & 0 & 0 & 0 & 0.008 \\
J45 & 0 & 0 & 0 & 0 & 0 & 0 \\
Recession and Resection (in diopters, D) & & & & 0.464 \\
SE & 0.250 & 0.380 & 0.130 & 0.130 & 0 & 0 \\
J0 & 0 & -0.120 & -0.110 & 0 & 0 & 0.491 \\
J45 & 0 & -0.100 & 0 & 0 & 0 & 0.121 \\
\hline
\end{tabular}

Table 6. Median values of preoperative and postoperative mean corneal corneal power and astigmatism based on the topography

\begin{tabular}{lcccccc}
\hline & Preoperative & Day 1 & Week 1 & Week 2 & Week 4 & Week 8 \\
Recession & & & & & \\
Km & 42.750 & 42.650 & 42.900 & 42.850 & 42.650 & 42.950 \\
Cylinder (D) & -0.850 & -1.550 & -1.000 & -1.100 & -1.250 & -1.400 \\
Recession and Resection & & & & & 0.952 \\
Km & 43.450 & 43.750 & 43.400 & 43.500 & 43.400 & 43.550 \\
Cylinder (D) & -0.950 & -1.650 & -1.500 & -1.200 & -1.200 & -1.450 \\
\hline
\end{tabular}

